\title{
Real Time Traffic Light Controller Using Image Processing
}

\author{
N. Karthikeyan ${ }^{1 *}$, N Praveen Kumar ${ }^{2}$, R Priyanka ${ }^{3}$, R Sangavee ${ }^{4}$, A Suthin Raj ${ }^{5}$ \\ Assistant Professor, Department of Electronics and Communication, Velalar College of Engineering and Technology, \\ Erode, India ${ }^{1}$
}

Scholars, Department of Electronics and Communication, Velalar College of Engineering and Technology, Erode, India ${ }^{2,3,4,5}$

\begin{abstract}
The frequent traffic jams at major intersections calls for an effective management system. This paper suggests implementing a smart traffic controller by image processing using Raspberry pi module. A camera will be installed on the four sides to capture image sequences. The captured images will then be analyzed using digital image processing for vehicles intensity, and based on this intensity on the road, traffic light can be controlled. It has been customized to control the traffic system by giving sufficient time for each side, depending on the number of cars on each direction to give out a way for ITS [Intelligent Transport System].
\end{abstract}

Keywords: Automatic Traffic, Raspberry pi, Image Processing, ITS [Intelligent Transport System].

\section{INTRODUCTION}

In modern life, we have to face with many problems, one of which is traffic congestion becoming more dangerous day by day. As a result of increase in vehicle traffic, many problems emerged, for example, traffic accidents, traffic congestion and so on. Traffic congestion was a very difficult problem. As a result, many investigators have paid attention to ITS (Intelligent Transportation System), such as predicting traffic flow based on traffic monitoring at the traffic junctions to detect bottlenecks. Several approaches for ITS have been implemented over many decades. In this paper, we will discuss about smart traffic control by using image processing to find intensity vehicles. Vehicles intensity is important in calculating traffic congestion on the roads. The main objective for detecting intensity of vehicles from an Image is to develop a methodology for automatic intensity of vehicles. Our method does not use filter or reference images, it directly processes the captured image and give out the intensity, depending on which the time and signals can be automatically changed depending on the conditions of traffic.

\section{METHODOLOGY}

There are many methods of detecting vehicles on road such as motion monitoring, installing lasers on both sides of the road etc., which is tedious and involves a large number of hardware.

\section{EXISTING METHODS}

\section{A. Using IR Transmitters and Receivers}

IR pairs are placed on the opposite side. When we place an obstacle in front of the IR pair, IR receiver will not be able to receive the IR rays. From the signals received by the IR receiver vehicle density can be determined and it signals the traffic light.

\section{Limitations:}

- As infrared waves have Line-of-sight transmission, the transmission cannot take place through walls, doors etc. 


\section{International Journal of Advanced Research in Computer and Communication Engineering}

Vol. 8, Issue 2, February 2019

- Line-of -sight transmission is blocked by common materials such as people, animals and data rate transmission is lower than normal wired communication.

- IR sensors sometimes may absorb normal light also. As a result, traffic system works in improper way.

- IR sensors work only for fewer distances.

- We have to arrange IR sensors in accurate manner otherwise they may not detect the traffic density.

\section{B. Using Microcontrollers}

\section{Limitations:}

- It uses the pre-defined hardware, which is functioning according to the program that does not have the flexibility of modification on real time basis.

- Bulky computers are used and is costly

\section{PROPOSED SYSTEM FOR TRAFFIC CONTROL USING VEHICLE DENSITY}

This system proposes a new system for controlling the traffic light by image processing using raspberry pi module. A camera will be installed alongside the traffic light. It will capture image sequences. The image sequence will then be analyzed using digital image processing for vehicle intensity, and according to traffic conditions on the road, traffic light can be controlled.

\section{Hardware Module}

1. Raspberry Pi Device

2. LED Lights

3. Camera-720p

4. Micro-controller:-ARDINO UNO

5. TM1637 LED Display

\section{Software Module}

1. Raspbian OS.

2. Open CV

3. Arduino IDE

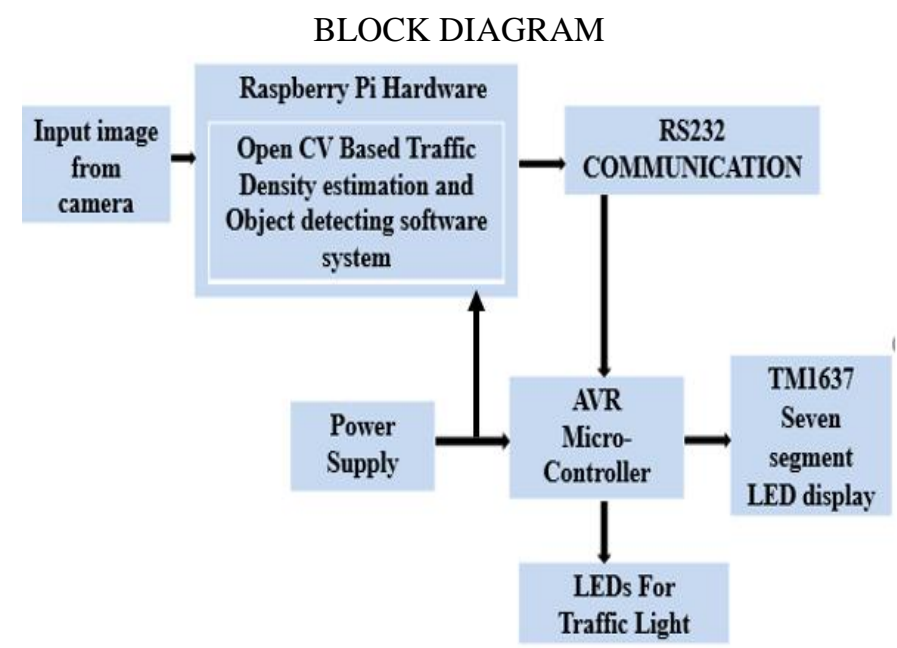

Fig 1: Block Diagram for Traffic control system 


\section{International Journal of Advanced Research in Computer and Communication Engineering}

Vol. 8, Issue 2, February 2019

\section{IMAGE PROCESING FOR VEHICLE INTENSITY}

Moving vehicle intensity is in the Image analysis. It can be used in many regions such as Image surveillance, traffic monitoring and people tracking. There are many motion segmentation techniques, like frame difference. Frame difference method has less computational complexity, and it is easy to implement; its difference between the current frame and the reference frame is above the threshold is considered as moving vehicle. Frame difference can detect the exact contour of the moving vehicle. This method gives result with an error up to $10 \%$.

\section{ARDUINO UNO MICRO CONTROLLER [ATMEL]}

Image processing of raspberry pi output signals are transmitted to Arduino Uno Micro Controller through RS232 Communication medium. Then depending upon Vehicle intensity higher priority of traffic in that lane is cleared through Light and Timing control system.

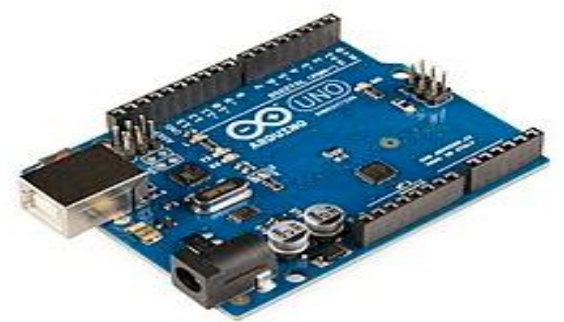

Fig 2: Arduino Uno Micro Controller

TM1637 LED DISPLAY

TM1637 is a chip for driving 7-segment displays. Based on intensity of vehicles highest and lowest priority of time will be display.

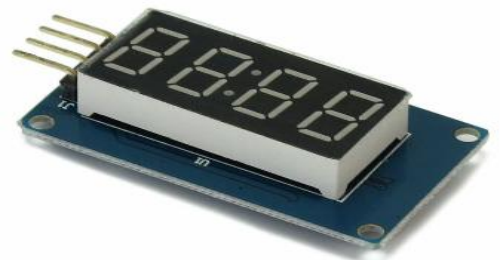

Fig 3: TM1637 LED Display

\section{TIMING AND LIGHT CONTROL BASED ON VEHICLE INTENSITY}

Experiments are carried out and depending upon the intensity of the traffic on the road we get the following results on time durations of various traffic lights.

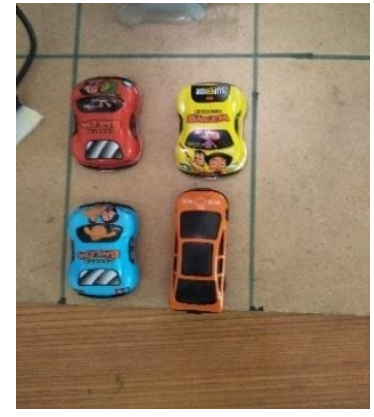

Fig 4a. Cars waiting under the focus of camera 1

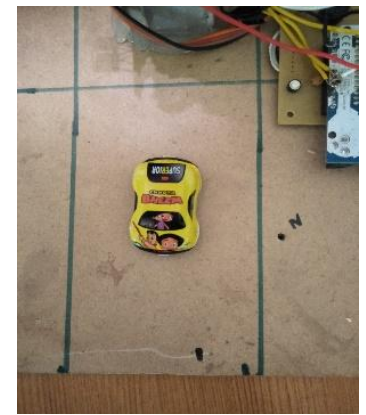

Fig 4 b. Cars waiting under the focus of camera 2 
Vol. 8, Issue 2, February 2019

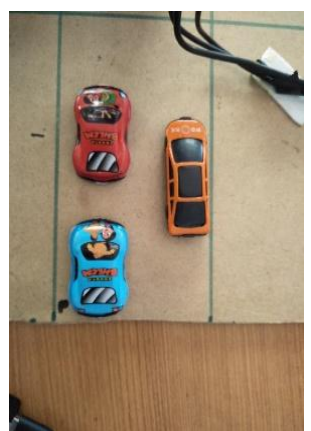

Fig 4c. Cars waiting under the focus of camera 3

After processing the images in raspberry pi the following result is obtained

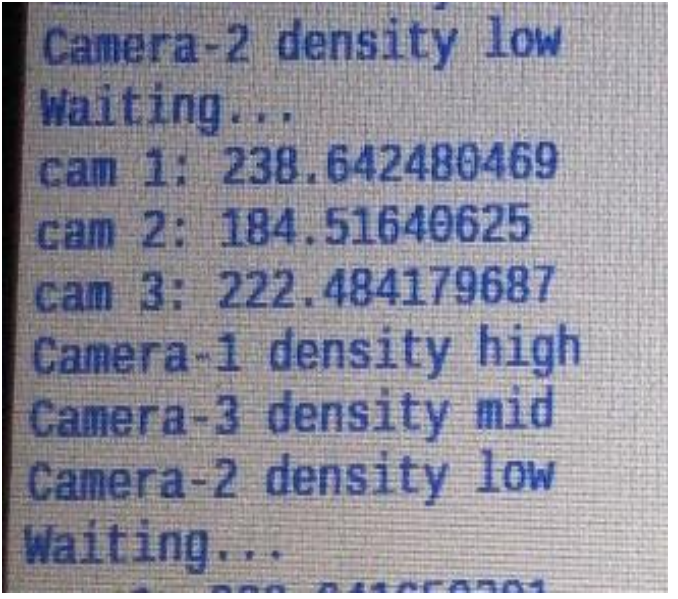

Fig 4.1 Each camera on the road side is ordered based on the density of the vehicles.

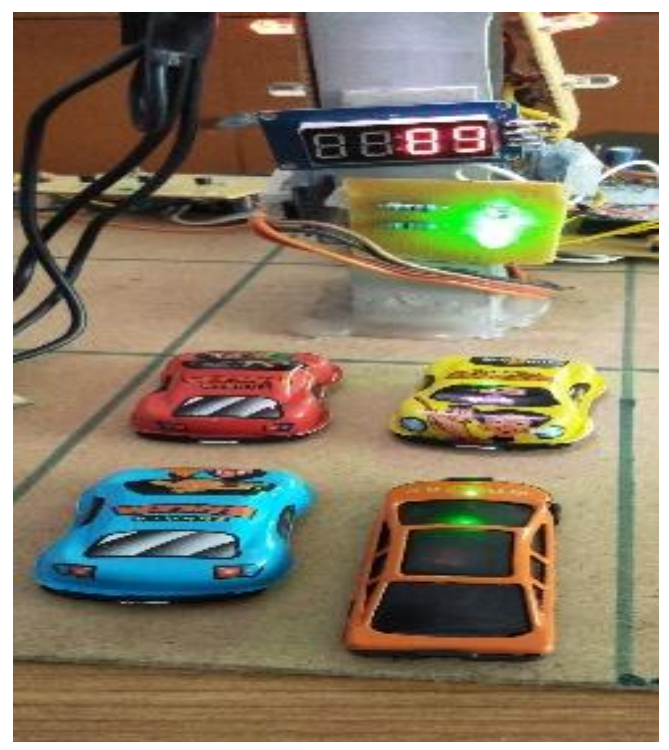

Result 1: Vehicle intensity high on camera 1 - GREEN light on for 90 seconds Result 2: Vehicle intensity low on camera 2 - GREEN light on for 40 seconds Result 3: Vehicle intensity moderate on camera 3- GREEN light on for 60 seconds 


\section{Advantage}

1. Compare to IR sensor it has high accuracy with fast response.

2 . Reduce the average stop time of vehicles.

\section{CONCLUSION}

In this paper discussed about the traffic light control in urban areas using image processing. It is used to control the traffic and providing the time for traffic signals based on traffic intensity. Overall, the system performance is good but it still needs improvement to achieve 100 percent accuracy.

\section{REFERENCES}

[1]. Saiba P A, Afeefa M U, Aruna T S, Clincy Jose, Radhika V M "Density Based Traffic Signal System using PIC Microcontroller "International Journal of Computer Trends and Technology (IJCTT) -Volume 47 Number 1 May 2017

[2]. Hossein rashid, Javad fazel, Mohsen Azizi, Mohammad rezza, "Intelligent Traffic Light Control Based on Clustering using Vehicular Ad-hoc Networks" IKT2015 7th International Conference on Information and Knowledge Technology 978-1-4673-7485-9/15/\$31.00 @2015 IEEE

[3]. Fig 4.2 Output for Cars waiting under the camera 1 